\title{
Estrategias para la implementación y adopción de componentes curriculares de ciberseguridad en programas de grado y posgrado
}

\author{
F.A. Corredor ${ }^{1}$, D. C. Franco ${ }^{2}$, J. E. Martínez ${ }^{3}$.
}

\begin{abstract}
Resumen - This paper presents the approach and application of strategies to assume two big problematic aspects of the cybersecurity sector, in which higher education institutions together with other stakeholders (productive sector and government) should cooperate and establish appropriate solutions. As a first aspect, the curricular design for integral training in cybersecurity is assumed, due to the density of themes, references of criteria and competences, which contrasts with the restrictions of space in the curricula of degree programs in information technologies (I.T.) with which you must address issues of foundation, deepening and emerging. moreover, the industry demand of the cybersecurity professionals, which currently exceeds supply; generating a global risk in organizations. The strategy is being implemented in a Colombian University, involving actively the student body to capture their interest in the area, as an objective of integral training and a graduated that generates labor supply.
\end{abstract}

Palabras Clave - Cybersecurity education, systems engineering, curricular strategies, undergraduate computing programs.

\section{INTRODUCCIÓN}

$\mathrm{L}$ A ciberseguridad es un aspecto que ha sido reconocido como elemento estratégico a nivel organizacional, abordado desde los diferentes estándares (CSF NIST, COBIT, ISO27001, ISO 27103, entre otros) y herramientas de software para gestión y operación específica, pero aunque hay cierto grado de consenso en los aspectos disciplinares propios de los contenidos tecnológicos, se genera cierto desacuerdo en los aspectos metodológicos y las temáticas a desarrollar, por las limitantes de espacio en los currículos de los programas profesionales de tecnologías de información (T.I.).

Desde la academia, se debe considerar esa gran cantidad de ejes temáticos y enfoques, así como la oferta de programas no formales y certificaciones, lo que dificulta garantizar la calidad y la secuencia organizada en ciclos y niveles de formación. Los diseños microcurriculares deben velar por desarrollar una gama de competencias (definidas por ABET, ABET CSAB, ACOFI, CSEC) las cuales deben cubrir aspectos generales y específicos de áreas fundamentales como la algoritmia y lógica, matemáticas, bases de datos, comunicaciones, etc. Pero también temas más avanzados que son de relevancia para los currículos de ingeniería de sistemas, informática o computación, como son la computación inteligente, big data y analítica, cloud computing, IoT y ciberseguridad, que conllevan a tomar decisiones de cobertura a nivel curricular para dejar componentes en el núcleo de formación - obligatorio - de las planes de estudio y dejar otros desde la oferta de electivas.

En el caso de ciberseguridad; se debe ser cuidadoso en la forma de estructurar y organizar la gran densidad de sus ejes temáticos, para responder al contexto local y global, pero también al proceso pedagógico que capte la atención de estudiantes, motivándolos hacia el área de la ciberseguridad (seguir formándose y ejercer laboralmente) y contribuir a la disminución del déficit de profesionales en ciberseguridad que sigue en aumento.

El artículo presenta los aspectos que definen el problema y propone una estrategia que está siendo adoptada en una universidad pública en Colombia, posteriormente una discusión de los aspectos encontrados y finalmente las conclusiones.

\section{CONTEXTo Del Problema}

La amplia dependencia tecnológica de las sociedades y sectores económicos ha venido presentando una preocupante situación de riesgos a nivel de ciberseguridad, donde el eslabón más débil sigue siendo el recurso humano interno de las organizaciones (menos del $40 \%$ de las organizaciones realiza capacitación y concienciación del personal [1]) y la principal amenaza la generan en su mayoría atacantes humanos externos $(69 \%$ de los ataques son generados por outsiders y el $52 \%$ de los ataques involucra operaciones de hacking [2]) lo cual dificulta las operaciones de protección y lo convierte en un problema que continúa en aumento. Esto ha generado la demanda de personal calificado para implementar una serie de factores de atención, gestión y operación, que garanticen su adecuado funcionamiento, dar cumplimiento a legislación específica y estar preparados ante las amenazas y vulnerabilidades que puedan materializar el riesgo. Esto acarrea varias situaciones de consideración para las instituciones de educación superior:

- El poco espacio de los currículos para asumir todos los temas emergentes y las competencias básicas, en el núcleo obligatorio de los planes de estudio. Lo que

\footnotetext{
${ }^{1}$ Felipe Andrés Corredor, M.Sc., Universidad de los Llanos, Grupo de Investigación GITECX, felcorredor@unillanos.edu.co.

${ }^{2}$ Diana Franco Mora, M.Sc., Universidad de los Llanos, Grupo de Investigación GITECX, dfranco@unillanos.edu.co.

${ }^{3}$ Javier Eduardo Martínez, Mg., Universidad de los Llanos, Grupo de Investigación GITECX, jmartinez@unillanos.edu.co.
} 
implica tomar decisiones y acciones respecto a las estrategias para formar integralmente a los nuevos profesionales con los conocimientos y capacidades suficientes en ciberseguridad.

- Incrementar la oferta de profesionales en ciberseguridad, ante la creciente demanda, que en la actualidad presenta un déficit de tres millones de profesionales de ciberseguridad en el mundo; lo que implica que deben plantearse estrategias (incluso extracurriculares) que incentiven el interés y agrado por esta disciplina.

En ambos casos, el sector educativo debe disponer de personal con amplio nivel de conocimiento teórico y aplicado, con visión analítica, crítica y conocimiento del contexto local y global. Lo cual conlleva a unir esfuerzos y capacidades de los profesionales del sector educativo con los profesionales del sector productivo [3]. Y aunque los tiempos de la academia son difíciles de sincronizar con los tiempos que maneja la industria, deben establecerse canales de cooperación y trabajo colaborativo que permitan mantener esa retroalimentación para los respectivos propósitos. (Formación, investigación y proyección social, así como la continuidad del negocio, innovación $\mathrm{y}$ oferta de servicios). Los proyectos de investigación, pasantías, cursos de formación bajo demanda, organización de eventos de concienciación, son algunas estrategias muy efectivas de propósitos comunes en ambos sectores.

En el primer aspecto, “diseño curricular” necesario, apropiado, y suficiente; las instituciones diseñan y adoptan una serie de estrategias para abordar una formación integral en ciberseguridad [4], en la cual se pueden definir y asumir los siguientes componentes:

- Teóricos: Matemáticas, probabilidad, estadística, grafos, teoría de la información, criptografía (sustitución, permutación, difusión, confusión, cifra simétrica en bloque y en flujo, cifra asimétrica, funciones hash, intercambio de clave, firma digital) y complejidad algorítmica.

- Tecnológicos: Programación, Sistemas operativos, sistemas distribuidos, Redes y comunicaciones, sistemas web y servicios telemáticos, arquitectura de software e infraestructura, etc. y sus respectivas habilidades tecnológicas.

- Organizacionales: Estándares y buenas prácticas de ISO, IEEE, NIST, OWASP, etc. Sistemas de gestión (procesos, riesgos, ciberseguridad), gobernanza de T.I., etc.

- Éticos y legales. Códigos de ética (Copnia - ley 842 de 2003, Código de ética de ACM), Ley 1273 de 2009, Ley 1928 de 2018 - Acuerdo de Budapest, Ley 1581 de 2012 (Protección de dato personales), Ley 1712 de 2014 (Transparencia de la información pública).

- Habilidades blandas: liderazgo, trabajo en equipo, comunicación verbal y escrita, argumentación, resolución de problemas, gestión del tiempo, etc. [5]

En una amplia gama de enfoques que provee la disciplina de ciberseguridad se hace necesario estructurar una secuencia adecuada de ejes temáticos en diferentes niveles de formación profesional y tomando como referentes los lineamientos curriculares internacionales, provistos por ACM-IEEE desde los enfoques de la computación, pero principalmente desde la disciplina de la ciberseguridad establecida en el CSEC 2017 Cybersecurity "Curriculum Guidelines for Post-Secondary Degree Programs in Cybersecurity", que establece las características de un programa de ciberseguridad y sus componentes estructurales del currículo como son : seguridad de datos, seguridad de software, seguridad de componentes, seguridad en conexiones, seguridad en sistemas y seguridad en el recurso humano [6]. Lo cual es completamente compatible con la perspectiva asumida en cada uno de los elementos planteados anteriormente para formación integral en el programa de ingeniería de sistemas de la Universidad de los Llanos; cuyo diseño se basó en un enfoque hibrido entre el lineamiento curricular en tecnologías de información TI.2017 [5] y Ciencias de la computación CS.2013 [7] de ACM-IEEE.

TABLA I.

\section{ASPECTOS CURRICULARES DE REFERENCIA, ACM-IEEE}

\begin{tabular}{|l|l|}
\hline \multicolumn{1}{|c|}{ Enfoque } & \multicolumn{1}{|c|}{ Áreas o aspectos de referencia } \\
\hline $\begin{array}{l}\text { Tecnologías de } \\
\text { Información. T.I. 2017 }\end{array}$ & $\begin{array}{l}\text { ITE-CSP Cybersecurity Principles (6\% del } \\
\text { currículo obligatorio) y ITS-CEC Cybersecurity } \\
\text { Emerging Challenges (4\% complementario) }\end{array}$ \\
\hline $\begin{array}{l}\text { Ciencias de la } \\
\text { computación. } \\
2013\end{array}$ & $\begin{array}{l}\text { IAS (Information Assurance and Security) Es } \\
\text { una de las 18 áreas de conocimiento en el núcleo } \\
\text { del plan de estudios; Dispone de seis horas en el } \\
\text { núcleo y 31,5 horas de 1AS distribuidas en otras } \\
\text { áreas. }\end{array}$ \\
\hline $\begin{array}{l}\text { Ciberseguridad. CSEC } \\
2017\end{array}$ & $\begin{array}{l}\text { Ciberseguridad como disciplina y áreas de la } \\
\text { Seguridad: datos, software, componentes, } \\
\text { conexiones, sistemas y humano, así como la } \\
\text { perspectiva de la industria. }\end{array}$ \\
\hline
\end{tabular}

La Universidad de los Llanos, desde la Facultad de Ciencias Básicas e Ingeniería cuenta con programas formales de grado y posgrado, así como de programas no formales como diplomados, en los cuales, se ha logrado introducir el componente de ciberseguridad, manteniendo una línea secuencial de temáticas y niveles de profundidad.

TABLA II.

CRÉDITOS EN CIBERSEGURIDAD EN PROGRAMAS DE T.I. EN LA UNIVERSIDAD DE LOS LLANOS

\begin{tabular}{|l|l|c|l|l|}
\hline Programa & Nivel & $\begin{array}{l}\text { Creiditos } \\
\text { total }\end{array}$ & $\begin{array}{l}\text { Créditos. } \\
\text { Relación. } \\
\text { Cibersegur }\end{array}$ & Aval MEN \\
\hline $\begin{array}{l}\text { Arquitectura } \\
\text { de software y } \\
\text { seguridad }\end{array}$ & $\begin{array}{l}\text { Diploma } \\
\text { do }\end{array}$ & 96 Horas & 48 Horas & $\begin{array}{l}\text { No requerido } \\
(2019)\end{array}$ \\
\hline $\begin{array}{l}\text { Ingeniería de } \\
\text { Sistemas }\end{array}$ & Grado & 165 & 8 & $\begin{array}{l}\text { Alta Calidad } \\
\text { (2016) por 6 } \\
\text { años. }\end{array}$ \\
\hline $\begin{array}{l}\text { Ingeniería } \\
\text { Electrónica }\end{array}$ & Grado & 169 & 4 & $\begin{array}{l}\text { Registro } \\
\text { Calificado } \\
\text { (2017) por 7 } \\
\text { años }\end{array}$ \\
\hline $\begin{array}{l}\text { Ingeniería de } \\
\text { Software }\end{array}$ & $\begin{array}{l}\text { Especial } \\
\text { ización }\end{array}$ & 28 & 3 & $\begin{array}{l}\text { Registro } \\
\text { calificado } \\
(2014) \text { por 7 } \\
\text { años. }\end{array}$ \\
\hline $\begin{array}{l}\text { Tecnologías } \\
\text { Digitales } \\
\text { emergentes }\end{array}$ & $\begin{array}{l}\text { Maestría } \\
\text { En } \\
\text { construc } \\
\text { ción. }\end{array}$ & 52 & 5 & $\begin{array}{l}\text { En } \\
\text { Construcción, } \\
\text { actualización al } \\
\text { decreto 1330 del } \\
\text { MEN }\end{array}$ \\
\hline
\end{tabular}

A nivel de pregrado, ciberseguridad se desarrollará a través de un curso obligatorio, nuevo, en séptimo semestre denominado "seguridad de la información" y un curso de profundización en décimo semestre denominado Curso de Profundización III - "aspectos avanzados de ciberseguridad" del área de teleinformática; plan de estudios que se encuentra acreditado 
de alta calidad por el MEN Colombia para un periodo de seis años.

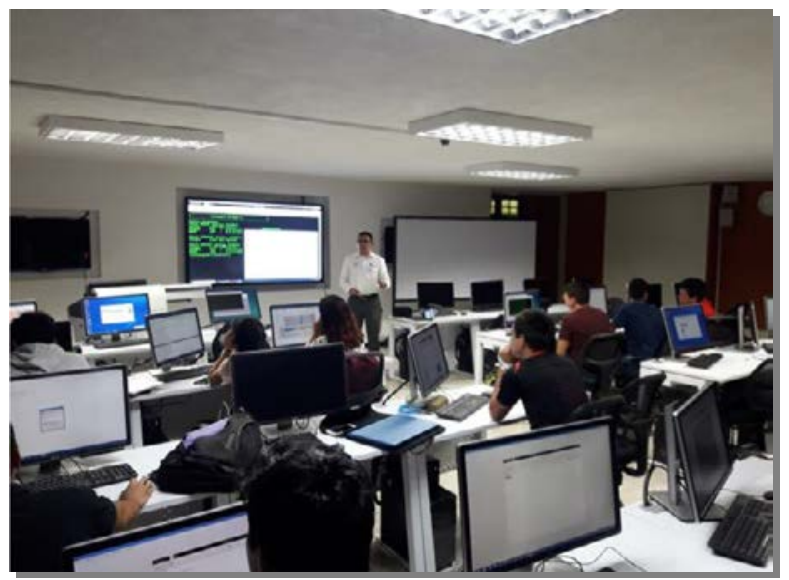

Figura 1. Estudiantes Décimo semestre, Curso de Seguridad de información, Ingeniería de sistemas.

A nivel de posgrado, desde la especialización en Ingeniería del software, se oferta una electiva denominada "ciberseguridad y hacking ético", la cual tiene un enfoque ofensivo, donde se presentan aspectos de gestión y se sigue un proceso metodológico de penetration testing (PTES - Penetration Testing Execution Estándar) [8], hasta la entrega de reporte y plan de acción. Así mismo se presentó una electiva en un programa de maestría con aspectos avanzados de criptografía y aplicaciones pasando por PKI y blockchain, hasta modelos de seguridad y arquitectura de seguridad. Estos cursos de posgrado pueden ser tomados por estudiantes de Ingeniería de sistemas como opción de grado.

A nivel de educación no formal, se diseñó y desarrolló un diplomado denominado "Arquitectura de software y seguridad", que está estructurado con el enfoque de seguridad para aplicaciones web, así como la arquitectura de infraestructura y la arquitectura de software, tomando como referente principal la guía de pruebas de OWASP 4.0 (OTG4) [9].

Para el segundo aspecto problemático, planteado en este artículo, que implica el déficit de profesionales en ciberseguridad, se requiere trabajar anticipadamente para que el estudiante mantenga el interés en la disciplina después del grado profesional, para ejercer en el área y continuar capacitándose (preferiblemente con incentivos financieros de apoyo). Diferentes organizaciones y el gobierno han determinado las situaciones de contexto que exponen varios de los aspectos problemáticos y las oportunidades que se tienen desde las instituciones que forman para la ciberseguridad:

El CONPES 3854 de 2016 que define "la política de seguridad digital - Colombiana" plantea la necesidad de formación para sus estudiantes en todos los niveles educativos y también para los profesores Universitarios, como se indica literalmente "Solicitar al Ministerio de Educación Nacional: a. Crear contenidos educativos complementarios relacionados con la gestión de riesgos de seguridad digital, y capacitar a: (i) los estudiantes de educación básica y media, (ii) los estudiantes de educación superior, y (iii) a los docentes" [10]; labor que han liderado desde el Ministerio de las TIC (MINTIC) y las Universidades Colombianas. Sin embargo, esto contrasta con los resultados emitidos por la encuesta nacional de seguridad informática de 2019 realizada por la asociación Colombiana de Ingenieros de sistemas (ACIS) a través de la cual concluye que solo el $28 \%$ de los empresarios reconoce que se están ofreciendo programas de grado y posgrado formales en ciberseguridad y el 31\% considera que no hay suficiente investigación en el área [11].

A esto se suma que a nivel mundial existe un gran déficit de profesionales en ciberseguridad, según lo plantea la Organización de Estados Americanos (OEA) y que afecta seriamente la adopción de su programa de ciberseguridad por parte de las organizaciones y los gobiernos del continente [12] y más crítico aún; lo planteado en el estudio de la fuerza laboral de Ciberseguridad de ISC, en el cual se determinó que el déficit ya se encuentra por el orden de tres millones de profesionales: "Una escasez de casi tres millones: este número puede parecer abstracto, pero está teniendo un impacto en el mundo real, en las empresas y en las personas que son responsables de su ciberseguridad. Según la encuesta, el $63 \%$ de los encuestados informan que sus organizaciones tienen escasez de personal de TI dedicado a la ciberseguridad. Y casi el $60 \%$ dice que sus empresas están en niveles moderados de riesgo extremo de ataques de ciberseguridad debido a esta escasez". [13]. En Asia se concentra el mayor déficit con 2,17 millones, luego Estados Unidos con cerca de medio millón y Latinoamérica con 0,2 millones. Para el caso de Colombia, el déficit se debe en gran parte a la normatividad que ha venido surgiendo en términos de protección de datos personales, ley de transparencia de la información pública y el CONPES 3854 de 2016, los cuales establecen nuevas obligaciones y condiciones para las organizaciones en torno a la seguridad de información.

Por esta razón las instituciones deben acompañar las iniciativas gubernamentales en torno al área y brindarle al estudiante insumos iniciales para permanecer explorando oportunidades como:

- Becas de formación posgradual: MINTIC, Colciencias - línea de investigación en ciberseguridad y ciberdefensa [14], Fulbright, Colfuturo, etc.

- Becas de cursos, eventos y certificaciones: MinTIC y OEA simposium, Cybersecurity women challenge, OWASP Latam Tour, Foros Universitarios en seguridad Digital (Universidad de los Andes).

- La Universidad de los Llanos brinda matrícula gratuita a cualquiera de sus programas de posgrado al mejor estudiante por facultad, que haya obtenido promedio de carrera igual o superior a 4.5. (Reglamento Estudiantil Universidad de los Llanos Acuerdo superior 015 de 2003, Artículo 68) [15].

- Cursos en posgrado como opción de grado de los programas de Ingeniería. Electivas de Ciberseguridad ofensiva, criptografía y gestión de seguridad, permiten que el estudiante de grado amplíe su perfil.

\section{DESARROLLO DE LA ESTRATEGIA}

Teniendo en cuenta que los estudiantes ingresan al programa de grado en edades que oscilan entre los 15 y 17 años de edad y su dedicación es presencial (tiempo completo), la estrategia se ha enfocado en dar continuidad al proceso de formación sobre los aspectos esenciales de ciberseguridad con un enfoque defensivo (protección) a nivel de grado como 
Ingeniero de sistemas; para que pueda posteriormente consolidar y continuar su proceso de formación avanzado en otros niveles de formación (Diplomado o posgrado) con un enfoque más ofensivo.

Como parte de este proceso, se diseñó y aplicó un instrumento de levantamiento de información a una muestra del 37\% (82 estudiantes de 219) de los estudiantes del programa de Ingeniería de sistemas de la Universidad de los Llanos, una universidad pública de orden nacional, lo que permitió evidenciar aspectos relacionados con el sector de interés para ejercer la profesión y la concienciación sobre las implicaciones de la ciberseguridad en su ámbito profesional, de igual manera permitió brindar a los funcionarios y entes colegiados, responsables del diseño curricular, elementos para la toma de decisiones sobre la adopción de los componentes de ciberseguridad en los programas académicos de grado y posgrado con sus respectivos planes de estudio.

También se revisó un resultado de autoevaluación del plan de estudios del programa de ingeniería de sistemas realizado en el año 2017, donde se relacionaron varias preguntas, que reflejan su grado de variación y permiten evaluar las estrategias tomadas en su momento.
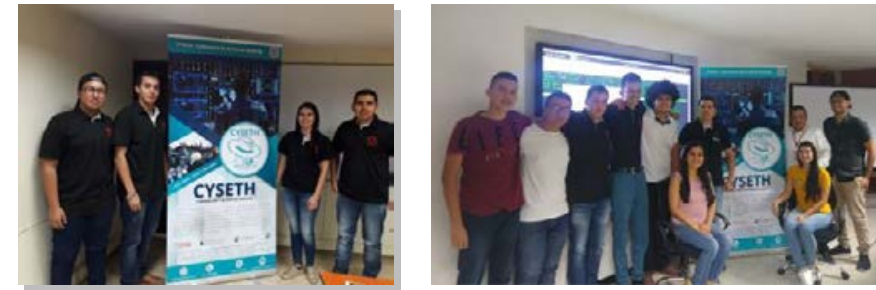

Figura 2. Semillero de Investigación CYSETH

Las acciones han sido tomadas desde un semillero de Investigación denominado CYSETH Unillanos (CyberSecurity \& Ethical Hacking Unillanos), conformado por cuatro profesores, 11 estudiantes (IV -VII semestre) y dos egresados, a partir de las siguientes estrategias adoptadas:

TABLA III.

ESTRATEGIAS Y RESULTADOS PLAN DE CIBERSEGURIDAD UNIVERSIDAD DE LOS LLANOS

\begin{tabular}{|c|c|}
\hline Estrategia & Resultados \\
\hline $\begin{array}{l}\text { 1. Apoyo a la oficina de } \\
\text { T.I. de la Universidad } \\
\text { (más de mil empleados } \\
\text { y siete mil estudiantes). } \\
\text { Diseño de políticas, } \\
\text { análisis de riesgos. }\end{array}$ & $\begin{array}{l}\text { Construcción de la política de } \\
\text { seguridad de información de la } \\
\text { Universidad, Apoyo a la } \\
\text { implementación de ISO } 27005 \text { e } \\
\text { ISO/IEC 27001 (en proceso). } \\
\text { Profesor auditor certificado } \\
\text { ISO27001 y estudiantes. }\end{array}$ \\
\hline $\begin{array}{l}\text { 2. Auditorías } r \\
\text { dependencias como } \\
\text { proyecto final de un } \\
\text { curso en décimo } \\
\text { semestre. }\end{array}$ & $\begin{array}{l}\text { Auditorías de controles de seguridad } \\
\text { en el marco de ISO/IEC 27001- } \\
27002 \text {, como proyecto final del curso } \\
\text { seguridad de la información en } \\
\text { décimo semestre, desde el año } 2015 \text { a } \\
\text { empresas de la ciudad (más de } 50 \\
\text { auditorías en los últimos } 4 \text { años). }\end{array}$ \\
\hline $\begin{array}{l}\text { 3. } \begin{array}{l}\text { Oferta de cursos en } \\
\text { posgrado }\end{array}\end{array}$ & $\begin{array}{l}\text { Oferta de dos cursos electivos de } \\
\text { seguridad ofensiva que se han } \\
\text { dictado en cinco cohortes. }\end{array}$ \\
\hline $\begin{array}{l}\text { 4. Diseño curricular en } \\
\text { pregrado y posgrado. }\end{array}$ & $\begin{array}{l}\text { Participación en el diseño de } 12 \\
\text { cursos que proveen requisitos para } \\
\text { asumir cursos de ciberseguridad. Así } \\
\text { como dos cursos específicos en } \\
\text { pregrado y dos en posgrado. }\end{array}$ \\
\hline Oferta de diplomados. & $\begin{array}{l}\text { Diseño y ejecución de un programa } \\
\text { de diplomado "Arquitectura de }\end{array}$ \\
\hline
\end{tabular}

\begin{tabular}{|c|c|c|}
\hline & & $\begin{array}{l}\text { software y seguridad". } 15 \text { estudiantes } \\
\text { certificados. }\end{array}$ \\
\hline & $\begin{array}{l}\text { Semillero } r \\
\text { investigación. (Apoyo } \\
\text { de estudiantes antiguos } \\
\text { para liderar los nuevos). }\end{array}$ & $\begin{array}{l}\text { Creación de un } \text { Semillero } \\
\text { denominado CYSETH, conformado } \\
\text { por } 11 \text { estudiantes y } 4 \text { profesores. } \\
\text { Vinculación de } 15 \text { estudiantes a } \\
\text { Proyectos de investigación de } \\
\text { GITECX (Grupo de Investigación } \\
\text { reconocido por Colciencias). }\end{array}$ \\
\hline & $\begin{array}{l}\text { Grupos de interés - } \\
\text { capítulo OWASP, rama } \\
\text { IEEE, Capitulo ACM. }\end{array}$ & $\begin{array}{l}\text { Búsqueda de apoyo y aval } \\
\text { institucional para iniciar la creación } \\
\text { del capítulo OWASP regional. Y } \\
\text { alianzas con otras iniciativas, que ya } \\
\text { existen en la Universidad. }\end{array}$ \\
\hline & $\begin{array}{lr}\text { Conferencias } & \text { y } \\
\text { demostraciones de } & \text { Hacking } \\
\text { Ethical y } \\
\text { blockchain } & \text { en } \\
\text { diferentes semestres. } & \end{array}$ & $\begin{array}{l}5 \text { conferencias ofrecidas de forma } \\
\text { gratuita ( } 3 \text { internas y dos externas - } \\
\text { Universidad Cooperativa } \\
\text { Uniminuto) en ciberseguridad, } \\
\text { hacking ético, ataques a la } \\
\text { autenticación y blokchain. }\end{array}$ \\
\hline & $\begin{array}{l}\text { Eventos, retos e } \\
\text { invitados } \\
\text { especializados. }\end{array}$ & $\begin{array}{l}\text { Se realizó un foro regional de } \\
\text { ciberseguridad, un CTF y apoyo a } 4 \\
\text { congresos (CTA - Congreso de } \\
\text { tecnologías abiertas) donde se } \\
\text { incluye el tema de ciberseguridad } \\
\text { (conferencistas incluidos). }\end{array}$ \\
\hline & $\begin{array}{l}\text { Encuestas y análisis del } \\
\text { contexto. }\end{array}$ & $\begin{array}{l}\text { s Dos instrumentos diseñados y } \\
\text { aplicados a más de } 130 \text { estudiantes, } \\
\text { los cuales fueron aplicados en } 2017 \text { y } \\
2019\end{array}$ \\
\hline 11. & $\begin{array}{l}\text { Banco de proyectos } \\
\text { para Trabajo de grado. }\end{array}$ & $\begin{array}{l}9 \text { proyectos ejecutados desde el } \\
\text { grupo de investigación GITECX y } 7 \\
\text { proyectos asignados desde el } \\
\text { semillero CYSETH. }\end{array}$ \\
\hline
\end{tabular}

Se diseñó un instrumento de levantamiento de información que se aplicó de manera presencial a estudiantes del programa de ingeniería de sistemas, de todos los semestres, buscando indagar tanto por los aspectos propios del diseño curricular y de la perspectiva laboral.

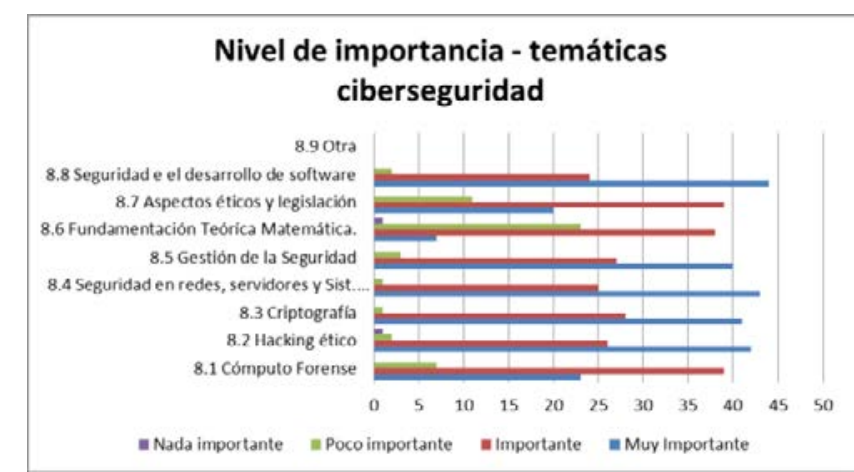

Figura 3. Resultados Preg. Nivel de importancia temática ciberseguridad.

En cuanto a lo curricular, se establecieron varios aspectos relacionados con la caracterización de los estudiantes, así como la importancia y necesidad de los temas en el plan de estudios. Por otro lado, también se indagaron aspectos específicos del agrado por las áreas y sectores del ejercicio profesional.

Muchos estudiantes son proclives al aprendizaje a través de lo praxiológico y manifestaron una preferencia por lo práctico $(59 \%)$ sobre lo teórico $(41 \%)$. Esto se puede relacionar con lo indagado sobre la relevancia de las temáticas que debe incluir un curso de ciberseguridad - ver figura 3 , encontrando que los 
temas asociados directamente con lo tecnológico (Hacking ético, criptografía y Seguridad en redes, servidores y sistemas operativos) son considerados por los estudiantes, como más importantes. Por otro lado, llama la atención que los fundamentos matemáticos, así como los elementos éticos y legales tengan una débil percepción de importancia, dentro de los cursos de seguridad; en el sentido de que la criptografía, catalogada como muy importante; requiere bastante fundamentación matemática y el hacking ético una sólida base ética y legal. De ahí la necesidad de que los profesores generen esta concienciación y orienten el diseño curricular ante estas dualidades.

Teniendo en cuenta que el instrumento aplicó preguntas con respuestas multidimensionales (cada tema con múltiples opciones de nivel de importancia), se calculó un valor total ponderado por cada temática de la siguiente manera:

\section{Total Ponderado $=\sum$ (Nivel Importancia* Factor Ponderación).}

Donde al nivel Muy Importante se le asignó un factor de 1 (100\%), al nivel Importante $0,7(70 \%)$ al poco Importante 0,3 $(30 \%)$ y al nada Importante $0(0 \%)$. Obteniendo los valores de la tabla 4 .

TABLA IV. RESULTADOS DE PONDERACIÓN TOTAL POR TEMAS.

\begin{tabular}{|c|c|c|c|c|c|}
\hline Temas & $\begin{array}{c}\text { Muy } \\
\text { Importan } \\
\text { te }\end{array}$ & $\begin{array}{c}\text { Important } \\
\text { e }\end{array}$ & $\begin{array}{c}\text { Poco } \\
\text { importante }\end{array}$ & $\begin{array}{c}\text { Nada } \\
\text { importante }\end{array}$ & $\begin{array}{c}\text { Total } \\
\text { Pondera } \\
\text { do }\end{array}$ \\
\hline $\begin{array}{c}\text { Cómputo } \\
\text { Forense }\end{array}$ & 23 & 39 & 7 & 0 & 60,3 \\
\hline Hacking ético & 42 & 26 & 2 & 1 & 71,2 \\
\hline Criptografía & 41 & 28 & 1 & 0 & 71,2 \\
\hline $\begin{array}{c}\text { Seguridad en } \\
\text { redes, servidores } \\
\text { y Sist. Operat. }\end{array}$ & 43 & 25 & 1 & 0 & 71,2 \\
\hline $\begin{array}{c}\text { Gestión de la } \\
\text { Seguridad }\end{array}$ & 40 & 27 & 3 & 0 & 69,9 \\
\hline $\begin{array}{c}\text { Fundamentación } \\
\text { Teórica } \\
\text { Matemática. }\end{array}$ & 7 & 38 & 23 & 1 & 48 \\
\hline $\begin{array}{c}\text { Aspectos éticos y } \\
\text { legislación }\end{array}$ & 20 & 39 & 11 & 0 & 57,5 \\
\hline $\begin{array}{c}\text { Seguridad e el } \\
\text { desarrollo de } \\
\text { software }\end{array}$ & 44 & 24 & 2 & 0 & 70,8 \\
\hline \begin{tabular}{c} 
Otra \\
\hline
\end{tabular} & 0 & 0 & 0 & 0 & 0 \\
\hline
\end{tabular}

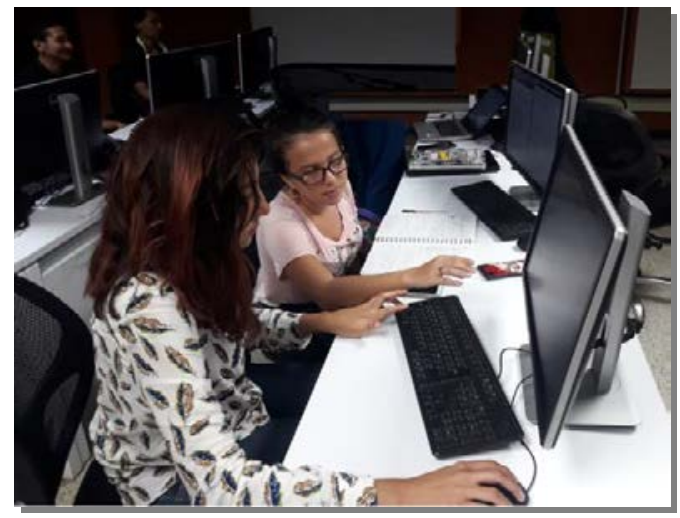

Figura 4. Estudiantes Décimo semestre, Curso de Seguridad de información, Ingeniería de sistemas.
Ante la diversidad de temas de impacto o emergentes que se deben asumir en los planes de estudio de los programas de T.I., es necesario determinar la percepción e interés, para enfocar los cursos de profundización y electivos en los diferentes niveles de formación. El estudio determinó que el $67 \%$ de los estudiantes considera que la "Ciberseguridad y hacking ético" junto a "Big Data" son los temas de mayor impacto y deben ser incluidos en el plan de estudios de manera preferencial, seguidos por "computación inteligente" con $65 \%$, como se ilustra en la figura 5 . Las Temáticas asociadas a la reutilización de componentes, educación virtual y Aplicaciones a la agroindustria no superan el $15 \%$ de interés.

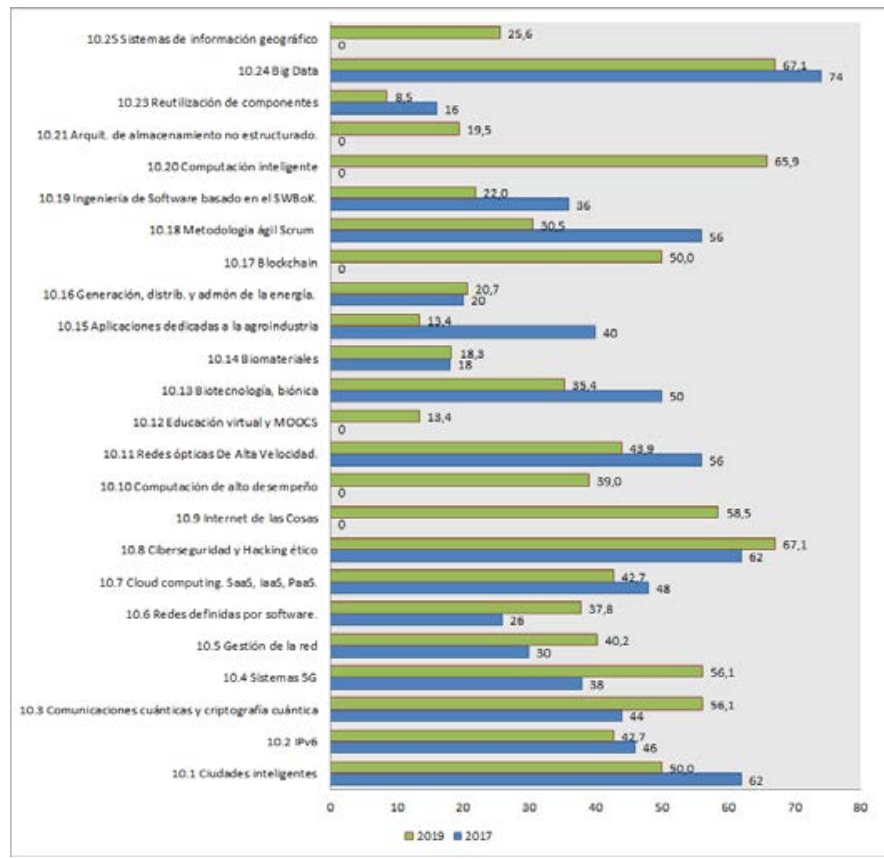

Figura 5.Resultados 2017 y 2019, Temas de impacto que deben incluirse en el plan de estudios

En lo referente contexto de la industria y el sector donde les interesa ejercer la profesión; el instrumento permitió determinar cómo los estudiantes perciben la incidencia de la adopción tecnológica y legislación (que impone ciertas condiciones), y su posible incidencia en la escogencia del sector de interés para ejercer la profesión.

El $74,4 \%$ de los estudiantes consideran que el comercio electrónico y las criptomonedas son el principal factor que incidirá en su ejercicio profesional como futuro Ingeniero de sistemas, de igual manera la normatividad del sector TIC $(73,2 \%)$, Incremento del uso de aplicaciones móviles $(63,4 \%)$ y el Incremento de los delitos y riesgos cibernéticos $(52,4 \%)$, según lo indicado en la figura 6 . 


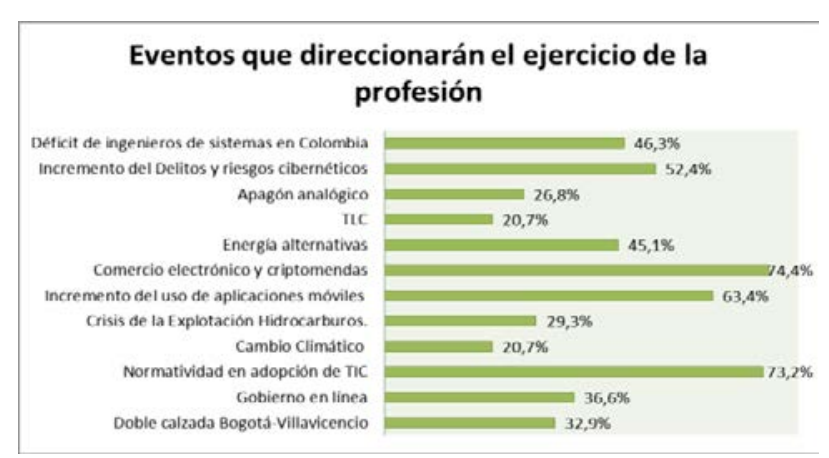

Figura 6. Resultados Percepción, eventos que direccionarán el ejercicio de la Ingeniería de sistemas.

Para la definición de los sectores indagados a los estudiantes, respecto a la preferencia para ejercer la profesión de ingeniero de sistemas, se tomaron los definidos por el MINTIC en el documento de caracterización de la industria TIC en Colombia [16], por lo cual se asumió el nombre "seguridad informática" literalmente, siendo el más afín a ciberseguridad.

Se consultó finalmente por 28 sectores de la industria T.I., encontrando que los sectores de mayor preferencia son Desarrollo y fabricación de software con el 73,1\%, seguido por seguridad informática con el $65,9 \%$ y Animación Digital/Video Juegos con el 62,2\%. Lo cual es coherente con las iniciativas que el MINTIC viene desarrollando específicamente en estos sectores.

\section{DISCUSIÓN}

Los parámetros del contexto, así como los temas relevantes y preferencias estudiantiles en el campo de las TIC, deben tener un seguimiento para identificar tendencias y las motivaciones sobre ellas.

Cuando se plantea formación integral, es esencial incluir en los currículos el componente ético y humanístico, lo cual también está determinado como criterio de acreditación para programas de ciberseguridad en ABET-CSAB (Cybersecurity and Similarly Named Computing Programs: criterio f. Seguridad Humana y criterio h. Seguridad social) [4], que a su vez se encuentran alineados con las guías curriculares de ACM-IEEE CSEC 2017 (Seguridad en el recurso humano Privacidad y seguridad en el Comportamiento social, Seguridad de Software - Ética, especialmente en el desarrollo, pruebas y revelación de vulnerabilidades).

Para el caso de la Universidad de los Llanos, se creó un nuevo curso en el plan de estudios denominado "ética y humanística" en séptimo semestre y se adopta un capítulo de ética y aspectos legales en todos los cursos relacionados con la ciberseguridad, lo cual evidencia un incremento del 54\% al $57 \%$ en la cantidad de estudiantes que consideran suficiente el componente "ético y humanístico" en el plan de estudios (Figura 7). Sin embargo se deben desarrollar nuevas estrategias para seguir incrementando este indicador que aún no es el ideal.

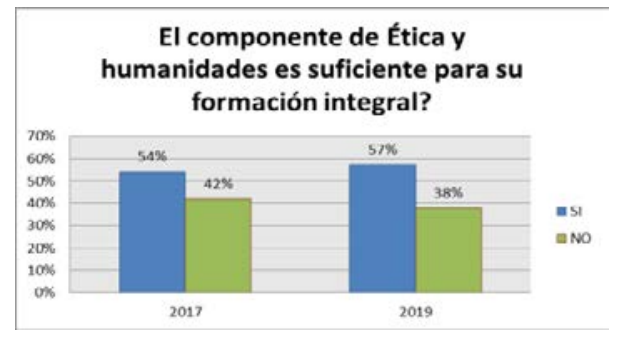

Figura 7. Comparación resultados Percepción estudiantes ética y humanidades.

Para el caso de los sectores de la industria de preferencia para ejercer la profesión, se retomó el estudio de autoevaluación de 2017 y se cruzaron los resultados de 2019 sobre los 16 sectores de la industria T.I (MINTIC) que habían sido indagados en ese momento; lo que permitió analizar tendencias y se evidenció (ver figura 8) que "de los cinco primeros sectores preferidos en el año 2017 (Big data, Ciberseguridad, metodología SCRUM, redes ópticas, ciudades inteligentes); todos descendieron en interés excepto ciberseguridad, que presentó un incrementó de 5.1\%", esto en parte debido a las actividades curriculares y extracurriculares promovidas por los actores interesados (profesores estudiantes y semilleros) en la Universidad.

De los 28 sectores, se incluyeron 12 sectores nuevos en el estudio actual; en los resultados, uno de ellos se ubicó en el primer lugar general (Desarrollo y fabricación de software) y otro en el tercero (animación digital/videojuegos), ambos con más del $60 \%$ de aceptación. Mientras que siete de estos sectores nuevos estuvieron por debajo del $20 \%$. Vale la pena resaltar que los sectores desarrollo de software y desarrollo de aplicaciones Web, son dos temas que han dinamizado de manera importante el área de la ciberseguridad a través del proyecto OWASP, que asume de manera directa estos aspectos con enfoque de seguridad y así mismo es una realidad de contexto, que ha presentado un aumento del $56 \%$ en los ataques web durante el 2018 [17].

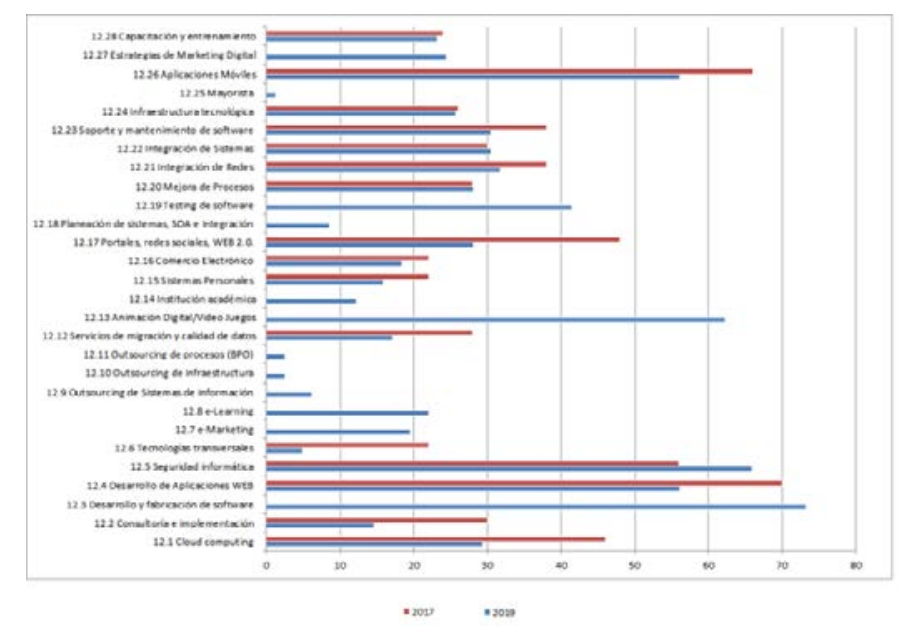

Figura 8. Sectores de la industria T.I. donde los estudiantes prefieren ejercer la profesión (Comparativa 2017 - 2019).

Al analizar los resultados de las preferencias de los sectores para ejercer la profesión y la percepción del grado de orientación que los eventos sociales y de gobierno puedan dar al sector de las TIC en Colombia, se procede a cruzarlas, y tratar de determinar posible incidencia mutua. 
En este sentido, la figura 9, presenta la correlación de eventos dinamizadores con los sectores de la industria T.I., donde el evento que más se considera dinamizador de la profesión sobre todos los sectores, es el "Comercio electrónico y criptomonedas" con $18 \%$ en promedio. Sin embargo, al relacionarlo con los dos sectores preferidos para ejercer laboralmente: "desarrollo y fabricación de software" presenta una incidencia de 56\% y "ciberseguridad y hacking ético" alcanza un $54 \%$ respectivamente. De igual manera el evento "delitos y riesgo cibernético" que tiene una relación directa con las dinámicas de la seguridad informática en 39\% como sector para ejercer la profesión, y $38 \%$ para desarrollo y fabricación de software.

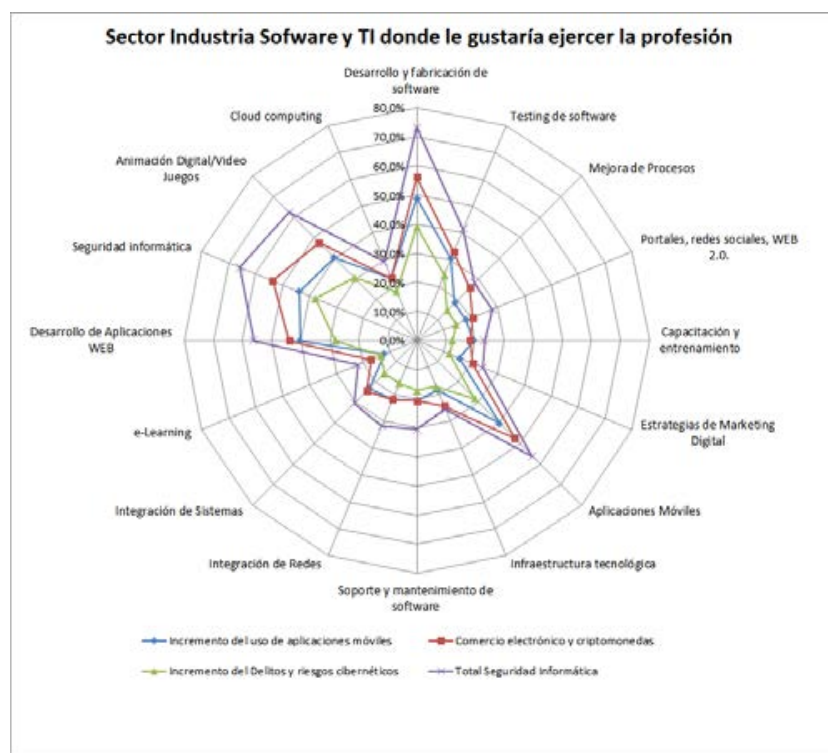

Figura 9. Correlación de Eventos del contexto TIC con las preferencias del sector para ejercer la profesión.

Otro aspecto que se viene promoviendo desde la OEA y ha encontrado eco en los gobiernos y organizaciones civiles, es la necesidad de brindar mayor inclusión a la mujer en el campo de la ciberseguridad. Para lo cual se han aplicado estrategias como el Cyberwomen Challenge en diferentes países (Colombia, Chile, Ecuador, Argentina, Estados Unidos), otorgando becas y recursos financieros. Sin embargo el problema no es solo para el campo de la ciberseguridad, sino de la ingeniería en general; para el caso particular de este proceso, la distribución porcentual de cantidad de estudiantes del programa de ingeniería de sistemas corresponde a $84.9 \%$ hombres y $15.06 \%$ mujeres y la población tomada para la muestra en este estudio, correspondió a $84 \%$ hombres y $16 \%$ mujeres.

En lo referente a preferencias de sector para el ejercicio profesional desde la perspectiva de género, el estudio determinó que del total de mujeres, el sector de Desarrollo y fabricación de software fue seleccionado por el 69,2\%, seguido por Animación Digital/Video Juegos con $61,5 \%$, Seguridad Informática $(61,8 \%)$ y aplicaciones móviles $(53,8 \%)$. El porcentaje del total de hombres que seleccionaron seguridad informática es de $68 \%$.
Sin embargo al observarlo desde el total de la población que escogieron cada sector, la representatividad de mujeres es reducida (ver figura 10): Desarrollo y fabricación de software fue $15 \%$, Animación Digital/Video Juegos con $10 \%$, seguridad informática (9\%) y aplicaciones móviles $(9 \%)$. Según el estudio de ISC [13], las mujeres representan el 24\% de la oferta laboral en ciberseguridad a nivel global, de ahí las iniciativas locales (Universitarias) y de la OEA, que muestran que a nivel local se tiene una brecha de 15 puntos porcentuales. .

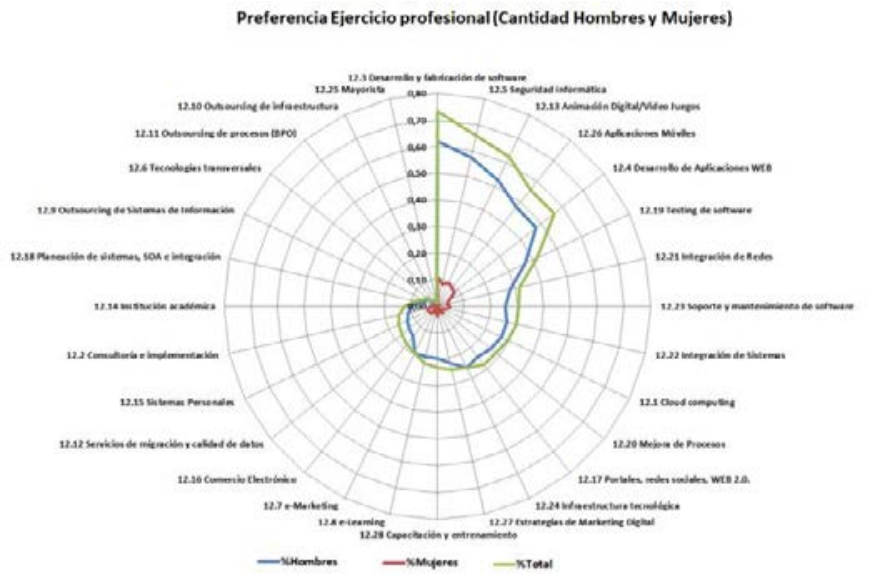

Figura 10. Proporción género - Preferencia sector para ejercer la profesión.

\section{CONCLUSIONES}

El diseño curricular debe responder a las necesidades del contexto local, sin desatender los aspectos globales; hay que tomar las múltiples referencias internacionales como un complemento a los enfoques de diseño propios y realizar los ajustes respectivos. Es de resaltar el gran aporte de los lineamientos establecidos en CSEC 2017, que puede ser visto como un documento de consenso, que integra en gran parte los esfuerzos de importantes organizaciones como ISC, ACM, IEEE y ABET entre otros.

La realización de proyectos de final de curso o carrera deben estar dirigidos al análisis y solución de problemas del contexto real, la realización de auditorías de seguridad en el marco de una estrategia pedagógica (proyecto de final de curso de profundización en Seguridad de Información en una empresa de la región) es un factor integrador entre la academia y la industria, que proyecta ampliamente al estudiante para continuar laborando o estudiando en el sector.

La mujer en ciberseguridad provee apenas el $24 \%$ de la capacidad laboral mundial pero a nivel local, solo representaría el 9\% de la capacidad laboral; por tal razón es una oportunidad con amplio margen de crecimiento para ampliar la oferta. Es necesario determinar de manera precisa a qué se debe el bajo índice de participación femenino y verlo como una oportunidad desde las iniciativas de la OEA y Ministerio de las TIC (MINTIC) para becas y financiación. 
Es importante conformar equipos de trabajo curricular que analicen y construyan todo un proyecto de formación en ciberseguridad, que trasciende un programa académico y un nivel de formación; desplegando competencias, temáticas y componentes curriculares en múltiples cursos de programas de grado, posgrado y no formal (virtual, MOOCS, curso libres). De la misma manera participar activamente en el diseño de los cursos que proveen los requisitos previos (como Sistemas Operativos, Redes, Programación, entre otros).

Promover el interés de los estudiantes involucrándolos en los procesos de construcción curricular con instrumentos de levantamiento de información, invitándolos a cursos y tallares abiertos, demostraciones en vivo y retos CTF (Capture The Flag), entre otros, por fuera de los espacios de clase y presión de la calificación, lo que permite conocer sus intereses particulares y grupales, para tenerlos en cuenta en las estrategias (Caso: Los estudiantes más antiguos, lideran y orientan a los más nuevos).

\section{AGRADECIMIENTOS}

Los autores expresan su gratitud a Dios y sus Familias, quienes siempre apoyaron este proceso. De igual manera, a la Universidad de los Llanos, al semillero de investigación CYSETH y al grupo de Investigación en tecnologías abiertas GITECX, por creer en este Proyecto.

\section{REFERENCIAS}

[1] CISCO Cybersecurity, "Anticipating the Unknowns: CISO Benchmark Study March 2019,” 2019.

[2] Verizon, "Verizon: 2019 Data Breach Investigations Report," 2019.

[3] R. K. Raj et al., "Perspectives on the future of cybersecurity education," Proc. - Front. Educ. Conf. FIE, vol. 2017Octob, pp. 1-2, 2017.

[4] ABET, "Computing Accreditation Commission CRITERIA FOR ACCREDITING COMPUTING PROGRAMS 2019$2020, " 2019$.

[5] C. S. I.-A. ACM, Information Technology Curricula 2017: Curriculum Guidelines for Baccalaureate Degree Programs in Information Technology. 2017.

[6] A. ACM, IEEE, CSEC - Curriculum Guidelines for PostSecondary Degree Programs in Cybersecurity, vol. Version 1., no. November. 2017.

[7] Computer Society IEEE - ACM, "CS2013: Computer Science Curricula 2013,” Computer, vol. 48, no. 3. 2013.

[8] "PTES Technical Guidelines - The Penetration Testing Execution Standard." [Online]. Available: http://www.pentest-

standard.org/index.php/PTES_Technical_Guidelines.
[Accessed: 30-Sep-2019].

[9] P. Leaders, M. Meucci, and A. Muller, "OWASP, Testing Guide 4.0," no. Cc.

[10] Departamento Nacional de Planeación, "Conpes 3854 Política Nacional De Seguridad Digital,” 2016.

[11] ACIS - Asociación Colombiana de Ingenieros de Sistemas, "XIX Encuesta Nacional de Seguridad Informática," Sistemas, vol. 151, pp. 12-41, 2019.

[12] O. Organization of American States, "Ciberseguridad marco NIST, Un abordaje integral de la Ciberseguridad," 2019.

[13] C. W. Study, "Cybersecurity Professionals Focus on Developing New Skills as Workforce Gap Widens Table of Contents," 2018.

[14] Colciencias, "Programas Nacionales de CTeI." [Online]. Available: https://www.colciencias.gov.co/investigadores/programasnacionales-ctei. [Accessed: 30-Sep-2019].

[15] Universidad de los Llanos, Reglamento estudiantil. 2003, p. 20.

[16] F. MinTIC, "Caracterizacion del sector teleinforatica, Software y TI en COlombia," 2015.

[17] Symantec, "ISTR Informe sobre las Amenazas para la Seguridad en Internet," 2019.

Felipe Andrés Corredor Magister en Software libre; del área de administración de redes y sistemas operativos. Especialista en Soluciones telemáticas e Ingeniero de Sistemas. Docente de planta e investigador de la Escuela de ingeniería de la Universidad de los Llanos. Sus áreas de desempeño son la seguridad informática y los sistemas distribuidos. Lidera el Grupo de investigación en Tecnologías abiertas GITECX, reconocido por Colciencias.

Diana Cristina Franco Mora Magister en Software libre; del área de administración de redes y sistemas operativos. Especialista en Soluciones telemáticas e Ingeniera de Sistemas. Docente de tiempo completo e investigadora de la Escuela de ingeniería de la Universidad de los Llanos. Sus áreas de desempeño son la teleinformática y el Software libre. Es integrante activa del Grupo de investigación en Tecnologías abiertas GITECX, reconocido por Colciencias.

Javier Eduardo Martínez Baquero, Magister en Tecnología educativa; Especialista en Instrumentación Industrial. Docente de planta e investigador de la Escuela de ingeniería de la Universidad de los Llanos. Director de la Especialización en Instrumentación y control industrial. Sus áreas de desempeño son la Automatización, Instrumentación. Es integrante activo del Grupo de investigación en Tecnologías abiertas GITECX, reconocido por Colciencias. 